\title{
IMPACT OF AN INTERACTIVE ANTI-SPEEDING THREAT APPEAL: HOW MUCH THREAT IS TOO MUCH?
}

Katarina Panić, Ghent University, Department of Communication Sciences, Belgium

Verolien Cauberghe, Ghent University, Department of Communication Sciences, Belgium

Patrick De Pelsmacker, University of Antwerp, Faculty of Applied Economics, Belgium

Corresponding author:

\section{Katarina Panić}

Ghent University - Department of Communication Sciences

Korte Meer 7-9-11

9000 Ghent

Belgium

Katarina.Panic@UGent.be

Tel: +3292646881

Fax: +3292646992 


\title{
IMPACT OF AN INTERACTIVE ANTI-SPEEDING THREAT APPEAL:
}

\section{HOW MUCH THREAT IS TOO MUCH?}

\begin{abstract}
This study investigates the impact of an interactive television public service announcement (PSA) containing an anti-speeding threat appeal on feelings of telepresence and behavioral intention. In a $2 \times 2 \times 2$ between-subjects factorial design with 213 participants, the level of threat evoked by a traditional PSA, by the interactive part of the PSA (dedicated advertising location) and by the preceding program context are manipulated to be either low or high. The results support the assumptions of the Extended Parallel Processing Model with regard to the effect of the level of perceived threat and perceived efficacy in an interactive media environment, and the important role of telepresence as a processing variable. The results of the three-way interaction effect of threat evoked by the program, the PSA, and the DAL on telepresence show that when the threat levels of the program and the PSA are both either low or high, exposure to the threatening information in the DAL does not generate a significantly higher feeling of telepresence. However, when a low-threat program is followed by a highthreat PSA, the threat level of the DAL has a positive effect on telepresence. The same trend is found with a high-threat program and a low-threat PSA, although the effect of the threat evoked by the DAL on telepresence is not significant at conventional levels. Finally, there is a positive effect of telepresence on the behavioral intention to reduce speeding which is partly mediated by the viewer's perceived efficacy to follow the recommended behaviour.
\end{abstract}




\section{Introduction}

Interactive digital television (IDTV) is a "new" medium that can be defined as the merging of the Internet and traditional television. Along with some potentially harmful consequences (e.g., viewers' ability to skip commercials), the increasing adoption and popularity of IDTV in many Western countries offers a lot of new opportunities for advertisers. One of the many new IDTV advertising formats is the microsite, which consists of a "30-second TV ad with a call-to-action button with clickable content or micro sites featuring individual still screens providing additional product information."1(p2) When the viewer clicks on the call-to-action button, he or she leaves the linear broadcast stream to enter a dedicated advertising location (DAL). There, he or she can navigate through the additional information, which can be structured in different layers. To avoid viewers missing part of their program while navigating in the DAL, the microsite format allows people to follow their program using the picture-inpicture technology. ${ }^{2}$ This implies that viewers can simultaneously watch the ongoing program in the upper-right corner while navigating through the information in the DAL.

For commercial goals, the microsite is promising. ${ }^{3}$ However, the question arises whether this new interactive advertising format can also be useful for public service announcements (PSAs). In the past, threat appeals have often been used in PSAs as an advertising strategy to influence people's attitudes and behaviors. ${ }^{4-7}$ Although a lot of threat appeal research is conducted in traditional print/television advertising, none of the previous studies have investigated the impact of threat appeals in interactive media.

The main research question of this study is whether an interactive DAL that follows a traditional 30-second PSA and a media context with both varying levels of evoked threat should either contain a low-threat message, or further increase the threat level to generate the highest persuasive impact? The current study tests the basic assumptions of the Extended 
Parallel Processing Model (EPPM) $)^{8,9}$ in an interactive environment and assesses the importance of telepresence (defined as "the sense of being present in the mediated environment" $)^{10(p 75)}$ as a processing variable. More particularly, we measure the impact of the level of threat in the PSA and in the media context on feelings of telepresence evoked in the DAL. In addition, we examine the influence of telepresence and the mediating impact of perceived response efficacy on behavioral intention.

\section{Conceptual Framework and Hypotheses Development}

\section{How threat appeals work: The Extended Parallel Processing Model}

Threat appeals are "persuasive messages designed to scare people by describing the terrible things that can happen to them if they do not do what the message recommends." ${ }^{\text {(p329) }}$ One of the most recent and comprehensive frameworks on how threat appeals work is the Extended Parallel Processing Model (EPPM). ${ }^{8,9}$ According to the EPPM, threat appeals trigger a process by which individuals first appraise the perceived severity of the threat. When the threat is not perceived as relevant or severe, people are not motivated to process the message in depth any further. Consequently, there is no response (i.e. no attitudinal or behavioral change) to the threat appeal. When the threat is perceived as sufficiently severe, feelings of fear may be elicited, which can further motivate people to process the message and eventually develop the intention to adopt the recommended behavior (danger control). However, when the levels of threat and fear are too high, people develop a defensive avoidance motivation to try to control the fear rather than the danger and as a result, they do not process the message in depth. This process is called fear control. ${ }^{8,11}$ 
According to the EPPM, after assessing the threat level of the message, individuals judge the perceived response efficacy of the message recommendation (the perception that the threat is reduced when the recommended behavior is adopted). The higher this perceived efficacy, the higher the probability that processing the message will lead to danger control. This implies that individuals will try to control the danger, resulting in message acceptance and adaptive behavior. When perceived efficacy is low, a person's feelings of fear are intensified by the perception that he or she is unable to avert the threat, especially when the threat is perceived as high. In this situation, a defensive avoidance mechanism (fear control) will again take place. In other words, threat appeals have a positive effect on adaptive responses only when the perceived efficacy to carry out the recommendation is high. ${ }^{12,13}$

\section{Telepresence as a threat appeal processing variable}

The ability to affect people's attitudes and behavior by exposing them to interactive threat appeals can be linked to the experience of telepresence. Telepresence is defined as "the perceptual illusion of non-mediation, produced by means of the disappearance of the medium from the conscious attention from the subject."14(p28) Traditionally, telepresence refers to immersive virtual environments. However, previous researchers have noted that telepresence is a useful construct for describing general human-computer interaction. ${ }^{15-18}$ Previous studies (e.g., Lombard et al., ${ }^{19}$ Lee, ${ }^{20}$ Ditton $^{21}$ ) even provided evidence that even traditional, analogue media such as magazines, movies and television (although not as immersive as, for instance, virtual reality environments) can induce a sense of telepresence. ${ }^{19,22,23}$ Kim and Biocca ${ }^{22}$ devoted a lot of attention to the generalization of telepresence in a traditional TV context. They state that the concept of telepresence is becoming an important component in our understanding of how people experience television. Hoffman \& Novak ${ }^{24}$ applied the concept of telepresence to an on-line environment. In the present study, we extrapolate and apply the 
telepresence construct to an interactive television environment, as this is a merger of traditional television and Internet.

Telepresence has two main antecedents, namely interactivity and vividness. ${ }^{10}$ Because the stimuli used in this study are both interactive (DAL) and vivid (audiovisual), telepresence can be expected to occur (see Coyle and Thorson) ${ }^{25}$. Witmer and Singer $^{26}$ report two additional conditions that are necessary for experiencing telepresence: immersion and involvement. As users focus their attention on the stimuli (due to vividness and interactivity) and feel highly involved with the threatening message, they will experience stronger feelings of telepresence. ${ }^{27}$ Indeed, Roser and Thompson ${ }^{28}$ find that exposing people to threat appeals results in a high involvement with the message. Also Cauberghe and collegues ${ }^{29}$ find that the persuasive effects of threat appeals are mediated by the involvement with the message. Given that threat appeals increase the involvement with the threat-evoking content ${ }^{16}$ and that the stimuli in this study are both vivid and interactive, we expect a positive impact of evoked threat on telepresence.

Using the innovations of IDTV, the current study evokes threat using multiple sources: the preceding program context (low vs. high threat), the traditional 30-second PSA (low vs. high threat) and the additional interactive information in the DAL (low vs. high threat). According to the main mechanisms of the EPPM, a low level of threat does not motivate people to further process the message. ${ }^{8}$ Therefore, a low-threat program followed by a low-threat PSA may not motivate viewers enough to thoroughly process the information in the DAL, regardless of the level of threat the DAL evokes. Consequently, we expect that threat evoked by the DAL will have no influence on the experience of telepresence in this situation.

When the level of evoked threat is very high, the EPPM predicts that people will also not be motivated to process the content of the message any further, because of defensive avoidance and fear control motivations. Therefore, when a high-threat program is followed by a high- 
threat PSA, viewers will not be motivated to control the danger, thereby losing their motivation to process the information in the DAL. In this case, the level of threat induced by the DAL will again have no effect on the experience of telepresence.

On the other hand, when a low-threat program is followed by a high-threat PSA or a highthreat program is followed by a low-threat PSA, threat levels may be sufficiently high to trigger processing of the message (cf. danger control), but not too high to lead to a defensive avoidance mechanism (cf. fear control). In this situation, we expect that presenting an additional strong-threat DAL will lead to a higher level of telepresence than a low-threat DAL. In other words, while we do not expect an effect of a high threat DAL on telepresence in case the program and PSA threat levels are both low or high, we do expect the following for the two other combinations of program context and PSA threat levels:

H1a: When the threat evoked by the program is low and the threat evoked by the PSA is high, a high level of threat evoked by the DAL has a more positive effect on telepresence than a low level of threat evoked by the DAL.

H1b: When the threat evoked by the program is high and the threat evoked by the PSA is low, a high level of threat evoked by the DAL has a more positive effect on telepresence than a low level of threat evoked by the DAL.

\section{Effect of telepresence on behavioral intention and the mediating role of perceived efficacy}

As argued in the previous section, we expect that exposing people to certain levels of an interactive threat appeal results in an experience of telepresence. An important variable to make individuals experience telepresence is involvement. Involvement can be defined as a dimension of telepresence ${ }^{30,31}$ or, according to others, an antecedent of telepresence (i.e., a necessary requirement for experiencing presence). ${ }^{26}$ Witmer and Singer ${ }^{26(\text { p227) }}$ define 
involvement as "a psychological state experienced as a consequence of focusing one's energy and attention on a coherent set of stimuli or meaningfully related activities and events. Involvement depends on the degree of significance or meaning that the individual attaches to the stimuli, activities or events." According to Witmer and Singer, users become more involved in a virtual experience as they focus more attention on virtual stimuli, which leads to an increased sense of telepresence. Thus, a higher level of telepresence implies more involvement and vice versa. ${ }^{32}$ Because earlier studies show that the persuasive impact of threat appeals is driven by the involvement with the message, ${ }^{29}$ we expect a positive effect of telepresence on behavioral intention.

Based on the logic of Hoffman and Novak's flow model ${ }^{24}$ and the EPPM, the effect of perceived threat-induced telepresence on behavioral intention is likely to be mediated by a person's perceived response efficacy pertaining to the threat appeal. According to the flow model, telepresence is an important antecedent of flow. The latter in turn triggers the perception of a higher behavioral control. Following the EPPM, this increased behavioral control will lead to a stronger intention to adopt the recommended behavior of the threat appeal.

First of all, telepresence is one of the primary antecendents of flow. ${ }^{24}$ Flow can be described as an intrinsically motivated optimal enjoyable mental state in which people experience a loss of self-consciousness, time and place. ${ }^{33}$ More telepresence therefore leads to more flow. Second, according to Hoffman and Novak, a stronger feeling of flow results in an increased perceived behavioral control (defined as "the perceived ease or difficulty of performing a behavior." $)^{34(\mathrm{p} 665)}$ Because flow is enhanced by telepresence, it can therefore be concluded that a sense of perceived behavioral control (indirectly) follows from telepresence.

Finally, the EPPM states that people who experience a higher perceived efficacy will have a higher intention to change their current behavior than people with a lower perceived efficacy. 
Also, Hoffman and Novak state in their flow model that perceived behavioral control directly affects behavioral intention and actual behavior. Since perceived behavioral control and perceived efficacy are conceptually similar, ${ }^{34}$ we expect an effect of telepresence on perceived efficacy and an effect of efficacy on behavioral intention. In other words, we hypothesize the following:

H2: The positive effect of telepresence on behavioral intention is (partly) mediated by perceived efficacy.

The conceptual model is presented in Figure 1.

$<$ Insert figure 1 here>

\section{Materials and Methods}

\section{Stimuli}

We used a 2 (threat of program context: low vs. high) $\times 2$ (threat of PSA: low vs. high) $\times 2$ (threat of DAL: low vs. high) between-subjects factorial design. Two PSA threat appeals were created, based on an existing anti-speeding PSA. The PSA showed young people leaving a party and getting into a car. They drive into a tunnel having fun but never come out again (suggesting that they had an accident). At the end of the spot, a silent frame is shown, stating: "Speeding causes accidents. Drive more slowly." This was the low-threat stimulus. For the high-threat stimulus, the PSA was manipulated by adding extra audiovisual features in a similar way to Potter et al. ${ }^{35}$ To make the PSA more threatening, we added the sound of a crashing car and an ambulance to the silent frame. Also, a picture showing a seriously 
wounded person in a hospital bed was added. In this high-threat condition, the message was "20 casualties per week. Drive more slowly." At the end of both PSAs, a red button and a call-to-action appeared on screen. By pressing this button, the respondents entered the DAL, in which they could navigate through the additional information about the dangers and risks of driving too fast.

In the high-threat DAL condition, the severity of the consequences presented in the text behind the click through link was high and the pictures included in the DAL were very vivid (e.g., showing seriously wounded victims and severe car accidents). Previous research has also used vividness to manipulate perceived threat (e.g., Meijnders et al. ${ }^{36}$ In the low-threat condition, the consequences of speeding were presented as less severe, and less vivid pictures were used.

In addition, we selected two different media contexts which were thematically congruent with the anti-speeding message. The high-threat context showed a movie excerpt (The Descent) with explicit images of people in a serious car accident causing critical injuries. The lowthreat condition came from the movie Taxi 2 and showed a scene in which many cars crash into one another, but without serious consequences for the drivers.

\section{Procedure and participants}

The study was conducted as an online survey. The respondents, who were recruited through a market research agency, all received an e-mail containing a link to the stimulus and the questionnaire. To avoid confounding effects, no indication was given about the aim of the study. The respondents were randomly assigned to one of the eight conditions. The average cell size per condition was 27 , and no cell had less than 20 observations, as recommended by Hair et al. ${ }^{37}$ After viewing the program for 10 minutes, the PSA appeared, containing a call-to- 
action button and a voice-over requesting the participants to press the red button to enter the DAL. Only the respondents who interacted with the PSA could fill in the questionnaire. In total, 213 valid responses were obtained, $50.7 \%$ of which were from male respondents. The respondents' age ranged from 20 to 57 years, with an average age of 41.6 years. The mean age of the male respondents did not differ significantly from the mean age of the female respondents $\left(M_{\text {male }}=41.8\right.$ vs. $\left.M_{\text {female }}=41.3 ; t(211)=.410, p=.682\right)$. In addition, the 8 experimental groups did not significantly differ in gender $\left(\operatorname{Chi}^{2}(1,7)=5.400, p=.611\right)$ nor in age $(\mathrm{F}(7,205)=.515, p=.823)$.

\section{Measures}

We measured the level of threat evoked by the PSA, the program and the DAL as experienced by the viewers using a five-item five-point Likert scale, based on the work of Laros and Steenkamp $^{38}$ (e.g., The message made me feel scared) (threat program: Cronbach's $\alpha=.891$; threat PSA: Cronbach's $\alpha=.897$; threat DAL: Cronbach's $\alpha=.915$ ). Telepresence was measured with Kim and Biocca's ${ }^{22}$ seven-item five-point Likert scale (e.g., While watching the program, I felt a new world was created; Cronbach's $\alpha=.875)$. This scale is a valid and reliable subscale of the Witmer and Singer $^{26}$ scale, developed to measure telepresence in a television environment. We measured behavioral intention with Putrevu and Lord's ${ }^{39}$ threeitem five-point scale (e.g., I will probably drive more slowly in the future; Cronbach's $\alpha=$ .744). Perceived efficacy was measured using Witte's six-item five-point Likert scale. ${ }^{8,9}$ This scale consists of two dimensions, namely self-efficacy (e.g., I can drive more slowly to avoid that I get involved in a car accident) and response efficacy (e.g., Driving more slowly is an effective way to avoid car accidents). However, an exploratory factor analysis using Varimax rotation showed that all six items of these two dimensions were highly correlated and loaded on one factor (variance explained $=67.68 \%$ ). Therefore, we combined the items into one 
overall perceived efficacy construct (Cronbach's $\alpha=.904)$. An overview of the measurement instruments is provided in Appendix 1. For each construct, we calculated the mean of the relevant items and used this measure for further analyses. Means, standard deviations, normality tests and Cronbach's alphas are summarized in Table 1.

<Insert Table 1 here>

\section{Results}

\section{Manipulation checks}

To assess the success of our manipulations, we measured the threat levels evoked by the PSA, the DAL and the program context to determine whether they were significantly different from each other. The results of the manipulation checks confirm that the levels of threat evoked by the program, the PSA and the DAL were adequately manipulated (program: $M_{\text {low threat }}=2.724$ vs. $M_{\text {high threat }}=3.261 ; \mathrm{t}(211)=4.719, p<.001 ;$ PSA: $M_{\text {low threat }}=2.703$ vs. $M_{\text {high threat }}=3.204$; $t(211)=4.854, p<.001 ;$ DAL: $M_{\text {low threat }}=2.825$ vs. $\left.M_{\text {high threat }}=3.262 ; t(211)=4.646\right)$, $p<.001)$. As indicated before, the DAL threat level was manipulated by, amongst others, the level of vividness of the DAL which significantly differs between the low and the high threat DAL $\left(M_{\text {low threat }}=3.161\right.$ vs. $\left.M_{\text {high threat }}=3.509 ; t(211)=11.818, p<.001\right)$.

\section{Analysis 1: effect on telepresence}

In the first analysis, we examined the variance in telepresence explained by the three independent variables: threat of the program, threat of the PSA and threat of the DAL. We analyzed the data using ANOVA. There appeared to be no main effects of threat evoked by 
the PSA $(F(1,212)=2.895, p=.090)$, threat evoked by the $\operatorname{DAL}(F(1,212)=1.324, p=.251)$, or threat evoked by the program context $(F(1,212)=.360, p=.549)$ on telepresence. However, the three-way interaction effect appeared to be marginally significant $(F(1,212)=3.603, p$ $=.059$ ). Simple effects tests further revealed the following (see Figures 2 and 3): as expected, a low-threat program followed by a low-threat PSA did not lead to a significant effect of the level of threat evoked by the DAL on the feelings of telepresence $\left(M_{\text {low threat }}=2.548\right.$ vs. $M_{\text {high }}$ threat $=2.550 ; t(56)=.007, p=.994)$. When a high-threat PSA follows a high-threat program, the threat evoked by the DAL had no effect on telepresence either, as expected $\left(M_{\text {low threat }}=\right.$ 2.746 vs. $\left.M_{\text {high threat }}=2.586 ; t(54)=.748, p=.457\right)$.

For a low-threat program followed by a high-threat PSA, the level of threat evoked by the DAL had a significant and positive effect on the feeling of telepresence $\left(M_{\text {low threat }}=2.769 \mathrm{vs}\right.$. $\left.M_{\text {high threat }}=3.177 ; t(50)=2.263, p=.028\right)($ see Figure 2$)$. When the level of evoked threat in the program was high (see Figure 3), the results show that, after respondents saw a low-threat PSA, the threat of the DAL had the expected effect on telepresence, although this effect did not reach conventional significance levels $\left(M_{\text {low threat }}=2.609\right.$ vs. $M_{\text {high threat }}=2.849 ; t(45)=$ 1.330, $p=.190)$. Thus, the findings support H1a, but not H1b.

<Insert Figures 2 and 3 here>

\section{Analysis 2: perceived efficacy as a mediator}

Next, we test the mediating effect of perceived efficacy on the relationship between telepresence and behavioral intention. Baron and Kenny ${ }^{40}$ propose a standard procedure for testing mediation. This procedure consists of three successive regression models. In Model 1, we test the direct effect of the independent variable (telepresence) on the outcome variable 
(behavioral intention). In Model 2, we test the effect of the independent variable (telepresence) on the potential mediator or intervening variable (perceived efficacy). Finally, in Model 3, we simultaneously estimate the effects of both the independent variable (telepresence) and the mediator (perceived efficacy) on the outcome variable (behavioral intention). In Model 3, if the mediator has a significant effect on the outcome variable and the impact of the independent variable disappears or is reduced, the effect of the independent variable on the outcome variable is fully or partly mediated by the mediator.

The results (see Table 2) show that telepresence has a significant effect on behavioral intention (Model 1) $(\beta=.323, p<.001)$. In Model 2, the effect of telepresence on perceived efficacy is positive and significant $(\beta=.218, p=.001)$. In Model 3, both telepresence and perceived efficacy were independent variables in a multiple regression, with behavioral intention as the dependent variable. The results show that both perceived efficacy $(\beta=.245, p$ $<.001)$ and telepresence $(\beta=.270, p<.001)$ have a significant effect on behavioral intention. However, when perceived efficacy is inserted in the multiple regression, the impact of telepresence on behavioral intention decreases (from $\beta=.323$ to $\beta=.270$ ), but remains significant. This indicates that the effect of telepresence on behavioral intention is partly mediated by perceived efficacy. In addition, the Sobel test $(z=2.672, p<.01)^{41}$ confirms that perceived efficacy significantly mediates the impact of telepresence on behavioral intention. Thus, the results support $\mathrm{H} 2$.

\section{Conclusions}


While most studies have investigated the impact of threat appeals in traditional television or print media, the current study focuses on the impact of threat appeals in an interactive television context.

The results of our study show that, depending on the level of threat induced by the PSA and the preceding program context, the level of threat evoked by the DAL has a different effect on the evoked feeling of telepresence. In turn, the feeling of telepresence has a positive effect on the intention to reduce speeding behavior, implying an adaptive danger-control process. This effect of telepresence on behavioral intention is partly mediated by perceived response efficacy, i.e. the perceptions of the ability to reduce one's speed and the beliefs about the effectiveness of reducing speed to avoid accidents.

Telepresence is a mental state in which a person feels physically present within the mediated environment and is absorbed by the mediated content. The results show that the positive effect of the level of threat evoked by the DAL on the feelings of telepresence is moderated by the threat evoked by the program and the PSA. Consistent with the EPPM, when the levels of threat evoked by both the program and the PSA are low, the general perceived threat level of the stimuli is too low to trigger motivation for further processing of any related information. Therefore, exposure to the additional threatening information in the DAL does not generate higher feelings of telepresence because under such circumstances, people are not motivated to process the information. On the other hand, high levels of threat evoked by both the program and the PSA lead to a general accumulated level of experienced threat that is so high that it produces a defensive motivation and triggers a fear control mechanism. ${ }^{6}$ In other words, the viewer does not want to process the additional information in depth due to a level of threat that is too high, leading to a relatively low experience of telepresence, regardless of the DAL threat level. Again, this is consistent with the EPPM. 
Lastly, in line with the expectations of the EPPM, the threat level of the DAL has a positive effect on telepresence when a low-threat program is followed by a high-threat PSA, generating a sufficient but not too high general level of threat, which triggers message processing. Although we found the same trend with a high-threat program followed by a lowthreat PSA, the effect of the threat level of the DAL on telepresence was not significant. There are several explanations for not finding a significant effect in this situation . First, the high threat evoked by the program may induce a transfer effect (cf. Affect Transfer Mechanism $^{42}$ and Excitation Transfer Mechanism ${ }^{43}$ ) of threat while watching a low threat PSA. However, this transfer may diminish in strength during low-threat PSA exposure, therefore decreasing the involvement and motivation when opening the DAL. Another explanation for not finding the expected result may be the nature of the threat appeal used in the high-threat program context. Compared with the other threat appeals, the threat appeal used in the program can be considered a shock message without a relief factor at the end (cf. Rossiter and Thornton). ${ }^{44}$ This might evoke a fear-control process, reducing the motivation to process the PSA and the DAL and therefore reducing the ability to experience feelings of telepresence.

Overall, a combination of very low or very high levels of threat evoked by the PSA and the program context is less effective in generating feelings of telepresence by a high-threat DAL than a medium level of threat evoked by the program and the PSA. In the moderate-threat conditions (more particularly a low-threat program followed by a high-threat PSA), a higher level of threat evoked by the DAL generates higher feelings of telepresence.

The feeling of telepresence also has a positive effect on the behavioral intention. This effect is partly mediated by the viewer's perceived response efficacy (cf. perceived behavioral control). This relationship between telepresence and antispeeding behavioral intention is mediated by the respondent's belief about whether the response (driving more slowly) would 
be effective in avoiding a car accident and by his or her perceived ability to perform this recommended response. We expected this mediating effect of perceived efficacy because feelings of telepresence simulate feelings of flow' which can increase a person's perceived

efficacy or perceived behavioral control..$^{25,34}$ In many models of threat appeals, and more particularly in the EPPM, perceived efficacy (cf. behavioral control) is an important driver of coping intention. ${ }^{8,9}$

\section{Managerial Implications}

In the past few years, the media landscape has changed a lot. The amount of new electronic media has increased significantly, and these new media have begun to take over traditional media. An example is the progressive substitution of traditional television by interactive television. As the media landscape changes, so does (marketing) communication. The business sector has embraced this alternative new medium as a new advertising medium. The current research shows that public health and other social marketing professionals can also gain valuable insights into how persuasion works in an interactive communication setting. Social marketers are now able to develop additional interactive information microsites following a traditional PSA. These microsites can evoke feelings of telepresence, which have a positive impact on behavioral change. In general, message effectiveness will be improved when the information in the DAL contains highly threatening information and is preceded by a program and a PSA which evoke a sufficient, but not extremely high level of combined threat. 


\section{Limitations and Further Research}

The limitations of the current study provide suggestions for further research. First, this study uses self-reported behavioral intention as the main dependent variable. Although prior research has found a positive and significant correlation between people's intention to speed and their actual behavior, ${ }^{45,46}$ future research should also measure speeding behavior through the use of a more real-life video simulator (cf. Walshe et al.). ${ }^{47}$

Second, neither the amount of clicks of the respondents nor the time and effort they spent interacting with the information in the DAL was recorded. These variables might be important because the more a person clicks on different information links, the longer a person is exposed to information, the more opportunity he or she has to process it, the more he or she will remember it, and the greater the impact on his or her attitude may be (e.g., Pieters and Bijmolt) ${ }^{48}$ Cauberghe and collegues ${ }^{49}$ find that the time spent in the DAL had an important mediating effect on brand recall and brand attitude. It is possible that there is also a mediating effect of time spent in the DAL on the relationship between amount of threat evoked by the DAL and the level of telepresence. Further research should incorporate these process variables. Additionally, in this study, the high threat PSA was a few seconds longer than the low threat PSA. This might have had a certain impact on how they were perceived. However, this is not a serious confound, since the main variable of interest - the evoked level of threat of the PSA - appeared to be correctly manipulated.

Third, respondents were requested to press the red button when it appeared on the television screen. Because of the respondents' inexperience with the new medium (i.e., IDTV) and the aim of the study to investigate the impact of the threatening DAL, this procedure was followed to avoid a large occurrence of non-response. Further research should however examine what motivates viewers to press (or not to press) the red button and whether the 4 
conditions (low-/high-threat program, low-/high-threat PSA) have an influence on the participants' motivation to enter the DAL.

Furthermore, it is possible that there are individual differences between people with respect to their ability to develop feelings of telepresence, just as for feelings of threat. ${ }^{50,51}$ A worthwhile research question might be whether telepresence can be considered a personality trait that varies across respondents and whether these individual characteristics have an effect on how people process interactive and/or threat appeals.

Finally, the topic of our study was anti-speeding behavior, a topic for which the perceived self-efficacy is expected to be rather high. Further research should examine the effect of threat appeals in an interactive context for different topics with varying levels of efficacy (e.g., addictive behavior such as drug abuse or smoking).

\section{Acknowledgements}

We thank the VAR (Vlaamse Audiovisuele Regie), Zappware, and InSites for their technical and operational support to accomplish this study.

\section{Author Disclosure Statement}

Competing financial interest: none declared for the submitted paper. 


\section{References}

1. Bellman S, Varan D. (2004) The impact of adding additional information to television advertising on elaboration, recall, persuasion. Paper presented at the 2004 ANZMAC Conference, Wellington, New Zealand.

2. Lekakos G, Papakiriakopoulos D, Chorianopoulos K. (2001) An integrated approach to interactive and personalized TV advertising. Proceedings of the 2001 Workshop on Personalization in Future TV.

3. Reading N, Bellman S, Varan D, Winzar H. Effectiveness of telescopic advertisements delivered by personal video recorders. Journal of Advertising Research 2006; June:217-227.

4. Tanner JF Jr, Hunt JB, Eppright DR. The protection motivation model: a normative model of fear appeals. Journal of Marketing 1991; 55:36-45.

5. Bennett R. Effects of horrific fear appeals on public attitudes towards AIDS. International Journal of Advertising 1996; 15:183-202.

6. Janis I. (1967) Effects of fear arousal on attitude change: Recent developments in theory and experimental research. In: Berkowitz L, ed. Advances in experimental social psychology, Vol. 3. New York: Academic Press, pp. 166-255.

7. Rogers R. (1983) Cognitive and physiological processes in fear appeals and attitude change: a revised theory of protection motivation. In: Cacioppo J, ed. Social psychophysiology. New York: Guilford Press, pp. 153-176.

8. Witte K. Putting the fear back into fear appeals: the Extended Parallel Process Model (EPPM). Communication Monographs 1992; 59:329-349. 
9. Witte K. Fear control and danger control: a test of the extended parallel process model (EPPM). Communication Monographs 1994; 61:113-134.

10. Steuer J. Defining virtual reality: dimensions determining telepresence. Journal of Communication 1992; 42:73-93.

11. Rippetoe PA, Rogers RW. Effects of components of protection-motivation theory on adaptive and maladaptive coping with a health threat. Journal of Personality and Social Psychology 1987; 52:596-604.

12. Blumberg SJ. Guarding against threatening HIV prevention messages: an information-processing model. Health Education and Behavior 2000; 27:780-795.

13. Snipes RL, LaTour MS, Bliss SJ. A model of the effects of self efficacy on the perceived ethicality and performance of fear appeals in advertising. Journal of Business Ethics 1999; 19:273-285.

14. Coelho CM, Tichon JG, Hine TJ, Wallis GM, Riva G. (2006) Media presence and inner presence: the sense of presence in virtual reality technologies. In: Riva G., Anguera MT, Wiederhold BK, et al, eds. From communication to presence: cognition, emotions and culture towards the ultimate communicative experience. Amsterdam: IOS Press, pp. 25-45.

15. Csikszentmihalyi M. (1990) Flow: the psychology of optimal experience. New York: Harper Row.

16. Ghani JA, Supnick R, Rooney P. (1991). The experience of flow in computermediated and in face-to-face groups. Proceedings of the twelfth international conference on Information systems. New York, United States, 229 - 237.

17. Trevino LK, Webster J. Flow in computer-mediated communication. Communication Research, 1992; 19:539-573. 
18. Webster J, Trevino LK, Ryan L. The dimensionality and correlates of flow in human-computer interactions. Computers in Human Behavior 1993; 9: 411-426.

19. Lombard M, Reich R, Grabe ME, Bracken CC, Ditton TB. Presence and television. The role of screen size. Human Communication Research 2000; 26:75-98.

20. Lee KM. Presence, explicated. Communication Theory 2004; 14:27-50.

21. Ditton T.B. (1997). The unintentional blending of direct experience and mediated experience: The role of enhanced versus limited television presentations. Unpublished doctoral dissertation. Temple University, Philadelphia, PA

22. Kim T, Biocca F. Telepresence via television: two dimensions of telepresence may have different connections to memory and persuasion. Journal of Computer Mediated Communication 1997; 3:1-24.

23. Bracken CC. Presence and image quality: the case of high-definition television. Media Psychology 2005; 7: 191-205.

24. Hoffman DL, Novak TP. Marketing in hypermedia computer-mediated environments: conceptual foundations. Journal of Marketing 1996; 50:50-68.Roser C, Thompson M. Fear appeals and formation of active publics. Journal of Communication 1995; 45:103-121.

25. Coyle JR, Thorson E. The effects of progressive levels of interactivity and vividness in Web marketing sites. Journal of Advertising 2001; 30:65-77.

26. Witmer BG, Singer MJ. Measuring presence in virtual environments: a presence questionnaire. Presence-Teleoperators and Virtual Environments 1998; 7:225-240.

27. Novak T, Hoffman D, Yung Y. Measuring the customer experience in online environments: a structural modeling approach. Marketing Science 2000; 19:22-42 
28. Roser C, Thompson M. Fear appeals and formation of active publics. Journal of Communication 1995; 45:103-121.

29. Cauberghe V, De Pelsmacker P, Janssens W, Dens N. Fear, threat and efficacy in threat appeals: Message involvement as a key mediator to message. Accident Analysis and Prevention 2009; 41:276-285.

30. Schubert T, Friedmann F, Regenbrecht H. (1999) Decomposing the sense of presence: factor analytic insights. Presented at Presence 1999-2 ${ }^{\text {nd }}$ International Workshop on Presence, University of Essex, Colchester, UK.

31. Lessiter J, Freeman J, Keogh E, Davidoff J. (2000) Development of a new crossmedia presence questionnaire: the ITC-sense of presence inventory. Presented at Presence $2000-3^{\text {rd }}$ International Workshop on Presence, Techniek Museum, Delft, the Netherlands.

32. Schubert T, Friedman F, Regenbrecht H. The experience of presence: factor analytic insights. Presence 2001; 10:266-281.

33. Csikszentmihalyi M, Lefevre J. Optimal experience in work and leisure. Journal of Personality and Social Psychology 1989; 56:815-822.

34. Ajzen I. Perceived behavioral control, self-efficacy, locus of control and the theory of planned behavior. Journal of Applied Social Psychology 2002; 32:665-683.

35. Potter R, LaTour M, Braun-LaTour K, Reichert T. The impact of program context on motivational system activation and subsequent effects on processing fear appeal. Journal of Advertising 2006; 35:67-80.

36. Meijnders A, Middenn C, Wilke H. Communications about environmental risks and risk-reducing behavior: the impact of fear on information processing. Journal of Applied Social Psychology 2006; 31:754-777. 
37. Hair JF, Tatham RL, Anderson RE, Black B. (1998). Multivariate Data Analysis, Fifth edition. Prentice Hall, p.342.

38. Laros F, Steenkamp JB. Importance of fear in the case of genetically modified food. Psychology \& Marketing 2004; 21:889-908.

39. Putrevu S, Tan J, Lord KR. Consumer responses to complex advertisements: the moderating role of need for cognition, knowledge and gender. Journal of Current Issues and Research in Advertising 2004; 26:9-24.

40. Baron RM, Kenny DA. The moderator-mediator variable distinction in social psychological research: conceptual, strategic and statistical considerations. Journal of Personality and Social Psychology 1986; 51:1173-1182.

41. Sobel ME. (1982) Asymptotic confidence intervals for indirect effects in structural equation models. In: S. Leinhardt, ed. Sociological methodology. Washington D.C.: American Sociological Association, pp. 290-312.

42. Coulter KS. The Effects of Affective Responses to Media Context on Advertising Evaluations. Journal of Advertising 1998; 27:41-51.

43. Tavassoli NT, Schutz CJ, Fitzsimons G.J. Program involvement: are moderate levels best for ad memory and attitude toward the ad? Journal of Advertising Research $1995 ; 35: 61-72$

44. Rossiter JR, Thornton J. Fear-pattern analysis supports the fear-drive model for antispeeding road-safety TV ads. Psychology \& Marketing 2004; 21:945-960.

45. Elliot B. (2001) The application of the theorists' workshop model of behavior change to motorists' speeding behavior in Western Australia, Unpublished Report. Western Australia, Office of Road Safety, Department of Transport. 
46. Vogel R, Rothengatter JA. (1984) Motives for speeding behavior on highways: an attitudinal study. Report VSC 84-09. Haren, The Netherlands: Traffic Research Center, University of Groningen.

47. Walshe D, Lewis E, Kim SI, O’Sullivan K, Wiederhold B. Exploring the use of computer games and virtual reality in exposure therapy for fear of driving following a motor vehicle accident. CyberPsychology \& Behavior 2003; 6:329-334.

48. Pieters R, Bijmolt T. Consumer memory for TV advertising: a field study of duration, serial position and competition. Journal of Marketing Research 1997; $23: 362-377$.

49. Cauberghe V, De Pelsmacker P. Opportunities and thresholds for advertising on interactive digital TV: a view from advertising professionals. Journal of Interactive Advertising 2006; 7. Available at: www.jiad.com. Accessed on December 8, 2006.

50. Wildavsky A, Dake K. Theories of risk perception: who fears what and why? Daedalus 1990; 119:41-60.

51. Gustafson PE. Gender differences in risk perception: theoretical and methodological perspectives. Risk Analysis 1998; 18:805-811.

Address reprint requests to:

Katarina Panić

Department of Communication Sciences, Ghent University

Korte Meer 7-9-11

9000 Ghent, Belgium

E-mail: Katarina.Panic@UGent.be 


\section{List of submitted figures and tables:}

- Figure 1: Conceptual model

- Figure2: Interaction effect between threat evoked by the PSA and the DAL on telepresence for a low-threat evoking program

- Figure 3: Interaction effect between threat evoked by the PSA and the DAL on telepresence for a high-threat evoking program

- Table 1: Descriptives of measurement instruments

- Table 2: Mediation analyses of perceived efficacy 
$\underline{\text { Figure 1: Conceptual model }}$

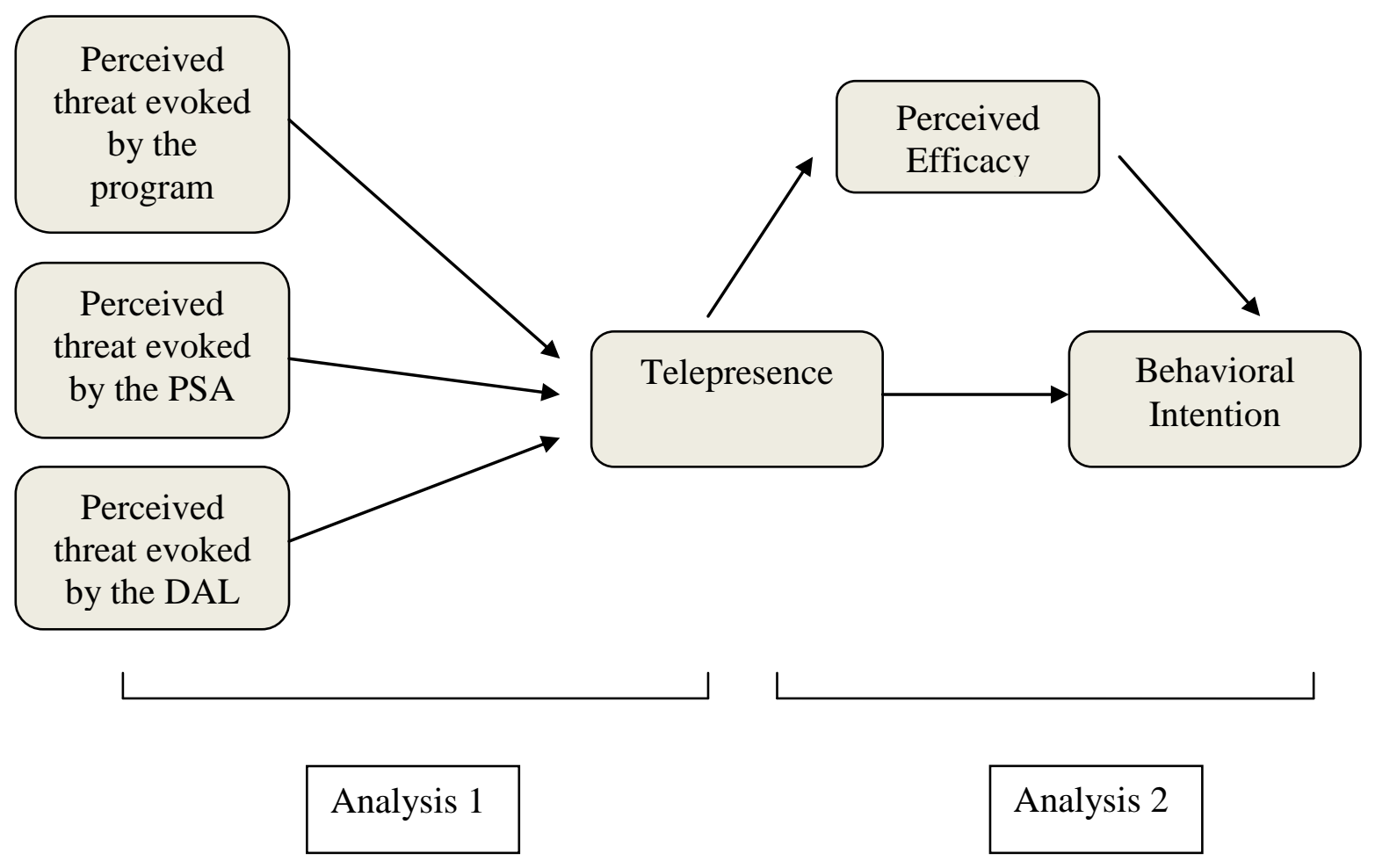


Figure 2: Interaction effect between threat evoked by the PSA and the DAL on telepresence for a low-threatevoking program

\section{Estimated Marginal Means of Telepresence}

Threat program is low

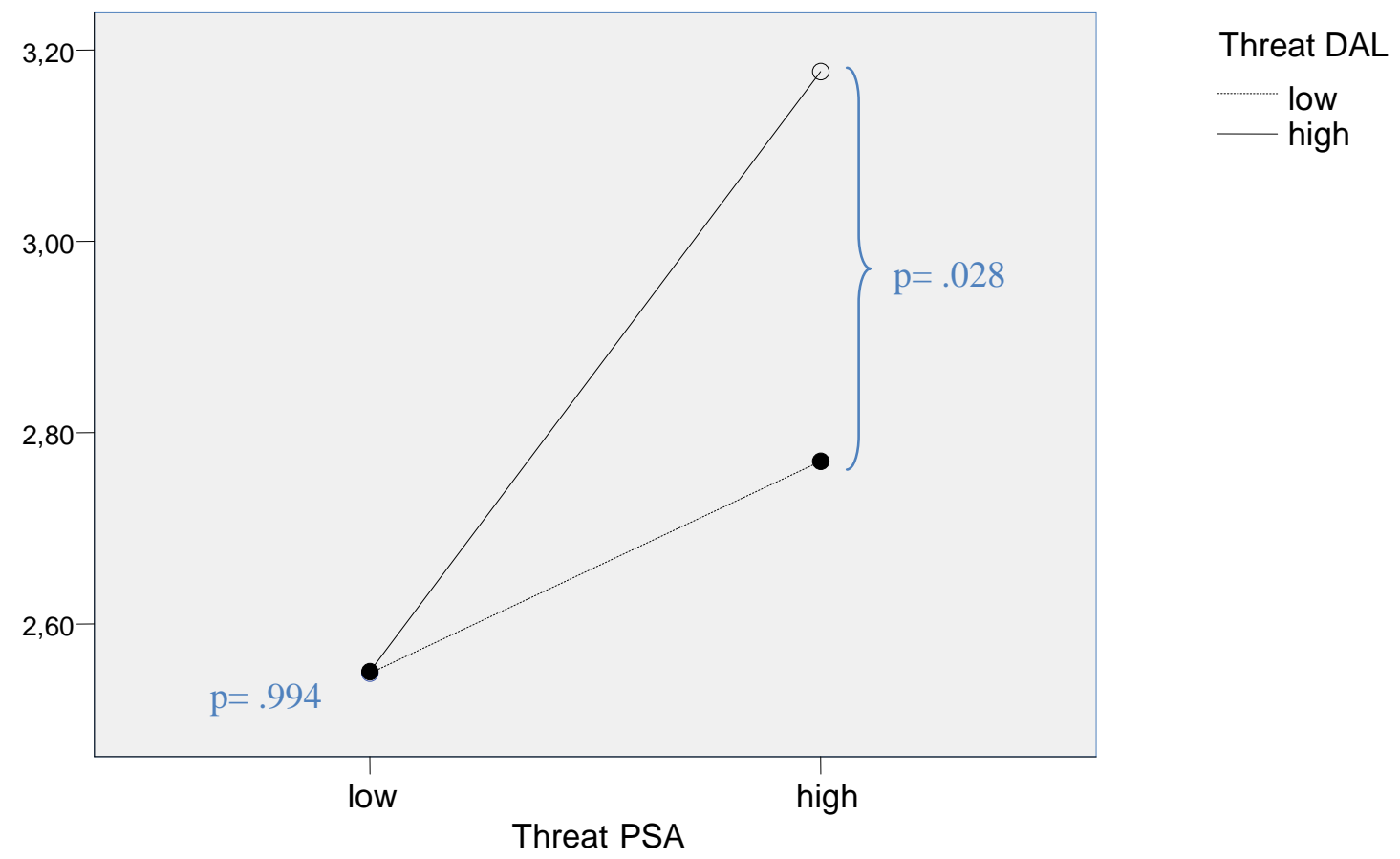


Figure 3: Interaction effect between threat evoked by the PSA and the DAL on telepresence for a high-threatevoking program

\section{Estimated Marginal Means of Telepresence}

Threat program is high

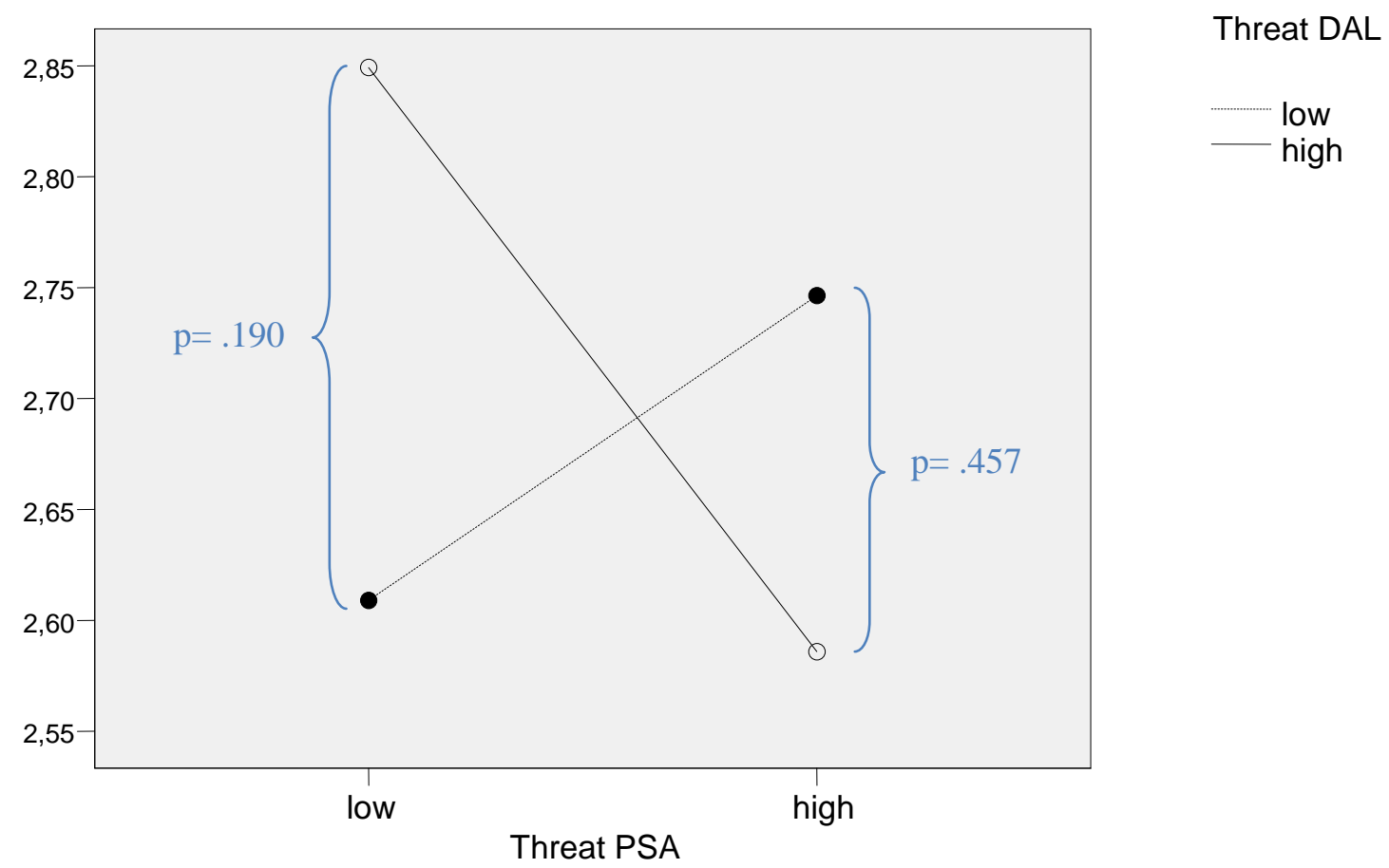




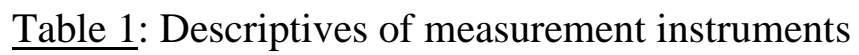

\begin{tabular}{|l|l|l|l|l|l|}
\hline & $\boldsymbol{M}$ & $\boldsymbol{S D}$ & Shapiro-Wilk & $\boldsymbol{p}$ & Cronbach's $\boldsymbol{\alpha}$ \\
& & & normality test & & \\
\hline Threat program & 2.983 & .876 & .971 & $<.001$ & .891 \\
\hline Threat PSA & 2.957 & .792 & .959 & $<.001$ & .897 \\
\hline Threat DAL & 3.041 & .718 & .927 & $<.001$ & .915 \\
\hline Telepresence & 2.726 & .773 & .960 & $<.001$ & .875 \\
\hline Behavioral Intention & 3.075 & .721 & .940 & $<.001$ & .744 \\
\hline Perceived Efficacy & 3.572 & .854 & .961 & $<.001$ & .904 \\
\hline
\end{tabular}


Table 2: Mediation analysis of perceived efficacy

\begin{tabular}{|c|c|c|c|c|c|c|}
\hline $\begin{array}{l}\text { Baron and } \\
\text { Kenny's } \\
\text { Steps }\end{array}$ & Relation & $R^{2}$ & 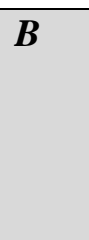 & $S E B$ & $\boldsymbol{\beta}$ & $p$ \\
\hline Step 1 & Telepresence $\rightarrow$ BI & .105 & .302 & .061 & .323 & $<.001$ \\
\hline Step 2 & Telepresence $\rightarrow$ Perceived Efficacy & .048 & .241 & .074 & .218 & .001 \\
\hline Step 3 & $\begin{array}{l}\text { Telepresence \& Perceived Efficacy } \rightarrow \text { BI } \\
\text { - Telepresence } \\
\text { - Perceived Efficacy }\end{array}$ & .162 & $\begin{array}{l}.252 \\
.207\end{array}$ & $\begin{array}{l}.060 \\
.055\end{array}$ & $\begin{array}{l}.270 \\
.245\end{array}$ & $\begin{array}{l}<.001 \\
<.001\end{array}$ \\
\hline
\end{tabular}




\section{Appendix 1}

1. The level of perceived threat was measured using a 5-item 5-point Likert-scale, ranging from I feel this emotion not at all (1) to I feel this emotion very strongly (5) (based on Laros and Steenkamp) $)^{38}$

The program/PSA/DAL made me feel ...

- Afraid

- Panicky

- Scared

- Worried

- Nervous

2. Telepresence was measured with Kim and Biocca's ${ }^{22}$ 7-item 5-point Likert-scale

- When I left the interactive part of the ad, I felt like I came back to the 'real world' after a journey. (strongly disagree/strongly agree)

- The interactive part of the ad created a new world for me. This world suddenly disappeared when I left the interactive part of the ad. (strongly disagree/strongly agree)

- While I was in the interactive part of the ad, I felt like a new World was created. (never/always)

- I sometimes forgot that I was in the middle of an experiment when I was in the interactive part of the ad. (never/ always)

- When I was in the interactive part of the spot, my body was in the room, but my mind was inside a new world, created by the spot. (never/always) 
- When I was in the interactive part of the spot, this created world seemed more real or present for me compared to the 'real' world. (never/always)

- The world that was created by the interactive part of the ad was only 'something I saw', rather than 'somewhere I visited'. (never/always)

3. Behavioral intention was measured with Putrevu and Lord's ${ }^{39} 3$-item 5-point Likert scale, ranging from strongly disagree (1) to strongly agree (5).

- I will probably not drive more slowly in the future.

- I will certainly not change my driving behavior.

- The next time I am in a car with friends, I will not encourage them to drive more slowly.

4. Perceived efficacy was measured with Witte's ${ }^{8,9}$ 6-item 5-point Likert scale, ranging from strongly disagree to strongly agree.

- Self-efficacy

- I can drive more slowly to avoid that I get involved in a car accident

- I have the ability to drive more slowly in order to avoid a car accident

- It is easy for me to drive more slowly to avoid a car accident this way

- $\underline{\text { Response efficacy }}$

- $\quad$ Driving more slowly is an effective way to avoid car accidents

- $\quad$ Driving more slowly helps to avoid car accidents

- If I drive more slowly, I have a smaller chance to be involved in a car accident 